\title{
3-MANIFOLDS WITH POSITIVE FLAT CONFORMAL STRUCTURE
}

\author{
REIKO AIYAMA AND KAZUO AKUTAGAWA
}

(Communicated by Lei $\mathrm{Ni}$ )

\begin{abstract}
In this paper, we consider a closed 3-manifold $M$ with flat conformal structure $C$. We will prove that if the Yamabe constant of $(M, C)$ is positive, then $(M, C)$ is Kleinian.
\end{abstract}

\section{Introduction And Main Theorem}

In 1988, Schoen and Yau [19] gave a final resolution for the Yamabe Problem (cf. [3, 15, 18]). In [19, Proposition 3.3], they also proved that any closed n-manifold with flat conformal structure of positive Yamabe constant is Kleinian, provided that $n \geq 4$. Moreover, under the assumption that an extended Positive Mass Theorem holds (but a proof has not yet appeared), they showed that the above assertion still holds even when $n=3$ (see [19, Proposition $\left.4.4^{\prime}\right]$ and the paragraph just before it). On the other hand, there are enormous examples of closed 3-manifolds with flat conformal structures which are not Kleinian (see [8, Remark 7.4]).

The purpose of this brief note is to prove the above assertion for the remaining case $n=3$.

Theorem 1.1. Let $M$ be a closed 3-manifold with flat conformal structure $C$. If its Yamabe constant is positive, then $(M, C)$ is Kleinian.

This assertion can be obtained by an argument in the proof of [1, the second assertion of Theorem 1.4], which is a combination of a result [19, Proposition 4.2], a positive mass theorem [1, the first assertion of Theorem 1.4] (different from the one Schoen and Yau mentioned in [19) and a classification of 3-manifolds with positive scalar curvature [7, 10, 11]. Here, we will explicitly give a proof of it (see also Remark 2.2 below).

The remaining sections are organized as follows. Section 2 contains some necessary definitions and preliminary geometric results. Section 3 is devoted to the proof of Theorem 1.1 .

\section{Preliminaries}

Let $M$ be a closed 3-manifold, that is, a compact 3-manifold without boundary. To simplify the presentation and the argument, we always assume that $\operatorname{dim} M=3$

Received by the editors April 5, 2011.

2010 Mathematics Subject Classification. Primary 53A30, 53C21; Secondary 57M10.

Key words and phrases. Differential geometry, geometric topology.

The second author was supported in part by the Grants-in-Aid for Scientific Research (C), Japan Society for the Promotion of Science, No. 21540097. 
throughout this paper. For each conformal class $C$ on $M$, the Yamabe constant $Y(M, C)$ of $(M, C)$ is defined by

$$
Y(M, C):=\inf _{g \in C} E(g), \quad E(g):=\frac{\int_{M} R_{g} d \mu_{g}}{\operatorname{Vol}_{g}(M)^{1 / 3}},
$$

where $R_{g}, \mu_{g}$ and $\operatorname{Vol}_{g}(M)$ denote respectively the scalar curvature, the volume element of $g$ and the volume of $(M, g)$. It is a finite-valued conformal invariant of $C$. The Yamabe constant $Y(M, C)$ is positive if and only if there exists a positive scalar curvature metric $g \in C$ (cf. [3]). A remarkable theorem [22, 20, 2, 17, 19] of Yamabe, Trudinger, Aubin and Schoen asserts that each conformal class $C$ contains a minimizer $\check{g}$ of $\left.E\right|_{C}$, called a Yamabe metric (or a solution of the Yamabe Problem), which is of constant scalar curvature

$$
R_{\check{g}}=Y(M, C) \cdot \operatorname{Vol}_{\check{g}}(M)^{-2 / 3} .
$$

Let $M_{\infty}$ be an infinite covering of $M$. We shall say that the fundamental group $\pi_{1}(M)$ of $M$ has a descending chain of finite index subgroups tending to $\pi_{1}\left(M_{\infty}\right)$ if it satisfies the following: There exists a family of subgroups $\left\{\Gamma_{i}\right\}_{i \geq 1}$ of $\pi_{1}(M)$ such that

(i) each $\Gamma_{i}$ is of finite index in $\pi_{1}(M)$ with $\Gamma_{i} \supset \pi_{1}\left(M_{\infty}\right)$,

(ii) $\pi_{1}(M)=\Gamma_{1} \supsetneq \Gamma_{2} \supsetneq \cdots \supsetneq \Gamma_{i} \supsetneq \Gamma_{i+1} \supsetneq \cdots$,

(iii) $\bigcap_{i=1}^{\infty} \Gamma_{i}=\pi_{1}\left(M_{\infty}\right)$.

Assume that $Y(M, C)>0$. Take a positive scalar curvature metric $g \in C$ and any point $p \in M$. Then, there exists the normalized Green's function $G_{p}$ for $L_{g}$ with a pole at $p$, that is,

$$
L_{g} G_{p}=c_{0} \cdot \delta_{p} \quad \text { on } M \text { and } \quad \lim _{q \rightarrow p} \operatorname{dist}(q, p) G_{p}(q)=1 .
$$

Here, $L_{g}:=-8 \Delta_{g}+R_{g}, c_{0}>0$ and $\delta_{p}$ stand respectively for the conformal Laplacian, a specific universal positive constant and the Dirac $\delta$-function at $p$. Assume also that the covering $P_{\infty}: M_{\infty} \rightarrow M$ is normal. Let $g_{\infty}$ denote the lift of $g$ to $M_{\infty}$, and $p_{\infty}$ a point in $M_{\infty}$ with $P_{\infty}\left(p_{\infty}\right)=p$. Then, there exists uniquely also a normalized minimal positive Green's function $G_{\infty}$ on $M_{\infty}$ for $L_{g_{\infty}}:=-8 \Delta_{g_{\infty}}+R_{g_{\infty}}$ with pole at $p_{\infty}$ (cf. [19]), which satisfies the following:

$$
\left(P_{\infty}\right)^{*} G_{p}=\sum_{\gamma \in \mathcal{G}} G_{\infty} \circ \gamma \quad \text { on } M_{\infty}
$$

Here, $\mathcal{G}$ stands for the group of deck transformations for the normal covering $M_{\infty} \rightarrow$ $M$. Set

$$
g_{\infty, A F}:=G_{\infty}^{4} \cdot g_{\infty} \quad \text { on } \quad M_{\infty}^{*}:=M_{\infty}-\left\{p_{\infty}\right\} .
$$

Then, $g_{\infty, A F}$ defines a scalar-flat, asymptotically flat metric on $M_{\infty}^{*}$ (cf. [15]). Note that this asymptotically flat 3 -manifold $\left(M_{\infty}^{*}, g_{\infty, A F}\right)$ has infinitely many singularities created by the ends of $M_{\infty}^{*}$. However, the mass $\mathfrak{m}_{A D M}\left(g_{\infty, A F}\right)$ of $\left(M_{\infty}^{*}, g_{\infty, A F}\right)$ can be defined in the usual way (cf. [4]). Note also that the positive mass theorem for asymptotically flat 3 -manifolds with singularities does not always hold (see [1, Remark 1.5-(2)] for instance).

Once this is understood, the following positive mass theorem holds as a special case of [1, the first assertion of Theorem 1.4]: 
Proposition 2.1. Let $(M, C)$ be a closed 3-manifold with $Y(M, C)>0$. Let $\left(M_{\infty}, g_{\infty}\right)$ be a normal infinite Riemannian covering of $(M, g)$ such that $\pi_{1}(M)$ has a descending chain of finite index subgroups tending to $\pi_{1}\left(M_{\infty}\right)$, where $g \in C$ is a positive scalar curvature metric and $g_{\infty}$ is its lift to $M_{\infty}$. For any point $p_{\infty} \in M_{\infty}$, let $G_{\infty}$ denote the normalized minimal positive Green's function on $M_{\infty}^{*}$ with pole at $p_{\infty}$. Then, the asymptotically flat 3-manifold $\left(M_{\infty}^{*}, g_{\infty, A F}\right)$ has nonnegative mass

$$
\mathfrak{m}_{A D M}\left(g_{\infty, A F}\right) \geq 0 .
$$

Remark 2.2. Assume that $M=\# \ell\left(S^{1} \times S^{2}\right)$ for $\ell \geq 2$ and $M_{\infty}$ is its universal covering. Note that $M_{\infty}$ is spin. For each small $\sigma>0$, consider the complete metric $g_{\sigma, A F}:=\left(G_{\infty}+\sigma\right)^{4} \cdot g_{\infty}$ with $R_{g_{\sigma, A F}} \geq 0$ on $M_{\infty}^{*}$ (cf. [19, Proposition 4.4']). Then, only one end of $\left(M_{\infty}^{*}, g_{\sigma, A F}\right)$ is asymptotically flat and the other infinitely many ends are merely complete. Gilles Carron and the referee kindly pointed out that Witten's approach [21] (cf. [16]) to the Positive Mass Theorem is still valid for the family $\left\{\left(M_{\infty}^{*}, g_{\sigma, A F}\right)\right\}_{0<\sigma<1}$. It implies that a more general positive mass theorem than Proposition 2.1] is a folk theorem for experts in this field, and Theorem 1.1 is too. But Proposition 2.1 itself is a complete form, and hence, by using it, we will give here an explicit and self-contained proof of Theorem 1.1.

A conformal 3-manifold $(M, C)$ is said to be locally conformally flat if, for any point $p \in M$, there exists a metric $\bar{g} \in C$ such that $\bar{g}$ is flat on some neighborhood of $p$. A conformal class $C$ on $M$ is called a flat conformal structure if $(M, C)$ is locally conformally flat. In [14, Kuiper proved that, for a simply connected locally conformally flat 3-manifold $\left(X, C^{\prime}\right)$, there is a conformal immersion into $\left(S^{3}, C_{0}\right)$ called a developing map, which is unique up to composition with a Möbius transformation of $\left(S^{3}, C_{0}\right)$. Therefore, the universal covering of a locally conformally flat manifold $(M, C)$ admits a developing map. Here, $\left(S^{3}, C_{0}\right)$ denotes the 3 -sphere $S^{3}$ with the conformal class $C_{0}:=\left[g_{0}\right]$ of the standard metric $g_{0}$ of constant curvature one. $(M, C)$ is called Kleinian if $(M, C)$ is conformal to $\Omega / \Gamma$ for some open set $\Omega$ of $S^{3}$ and some discrete subgroup $\Gamma$ of the conformal transformation group $\operatorname{Conf}\left(S^{3}, C_{0}\right)$, which leaves $\Omega$ invariant and acts freely and properly discontinuously on $\Omega$. Note that, if the developing map of the universal covering of a locally conformally flat manifold $(M, C)$ is injective, then $(M, C)$ is Kleinian.

With this understanding, the following criterion also holds as a special case of [19, Proposition 4.2]:

Proposition 2.3. Let $(M, C)$ be a closed 3-manifold with $Y(M, C)>0$, and $(\widetilde{M}, \widetilde{g})$ be the universal Riemannian covering of $(M, g)$, where $g \in C$ is a positive scalar curvature metric. For any point $\widetilde{p} \in \widetilde{M}$, let $\widetilde{G}$ denote the normalized minimal positive Green's function on $\widetilde{M}$ for $L_{\widetilde{g}}$ with pole at $\widetilde{p}$, and $\left(\widetilde{M}-\{\widetilde{p}\}, \widetilde{g}_{A F}=\widetilde{G}^{4} \cdot \widetilde{g}\right)$ the asymptotically flat 3-manifold as above. If the mass $\mathfrak{m}_{A D M}\left(\widetilde{g}_{A F}\right)$ is nonnegative, then the developing map of $(\widetilde{M},[\widetilde{g}])$ is injective. In particular, $(M, C)$ is Kleinian.

Remark 2.4. We remark that the mass $\mathfrak{m}_{A D M}\left(\widetilde{g}_{A F}\right)$ is equal to the ADM energy $E$ of $\left(\widetilde{M}-\{\widetilde{p}\}, \widetilde{g}_{A F}\right)$ appearing in [19, page 64$]$ up to a positive constant.

\section{Proof of main theorem}

Proof of Theorem 1.1. Consider the universal covering $\widetilde{M}$ of $M$ and denote the lift of the flat conformal structure $C$ by $\widetilde{C}$. If $\left|\pi_{1}(M)\right|<\infty$, then $(\widetilde{M}, \widetilde{C})$ is conformal 
to $\left(S^{3}, C_{0}\right)$ by Kuiper's Theorem [14. Hence, $(M, C)$ is Kleinian. From now on, we assume that $\left|\pi_{1}(M)\right|=\infty$, that is, the degree of the covering map $P: \widetilde{M} \rightarrow M$ is infinite.

Take a unit-volume Yamabe metric $g \in C$, and consider its lift $\widetilde{g} \in \widetilde{C}$ to $\widetilde{M}$. Note that $R_{\widetilde{g}}=R_{g}=Y(M, C)>0$. Take any base points $p \in M, \widetilde{p} \in \widetilde{M}$ satisfying $P(\widetilde{p})=p$, and fix them. Then, let $\widetilde{G}$ denote the normalized minimal positive Green function on $\widetilde{M}$ for $L_{\widetilde{g}}$ with pole at $\widetilde{p}$, and the mass $\mathfrak{m}_{\mathrm{ADM}}\left(\widetilde{g}_{A F}\right)$ of the asymptotically flat 3-manifold $\left(\widetilde{M}-\{\widetilde{p}\}, \widetilde{g}_{A F}:=\widetilde{G}^{4} \cdot \widetilde{g}\right)$.

Suppose that

$$
\mathfrak{m}_{\mathrm{ADM}}\left(\widetilde{g}_{A F}\right) \geq 0 .
$$

Recall that we can choose the base point $\widetilde{p} \in \widetilde{M}$ arbitrarily. It then follows from Proposition 2.3 that the developing map of $(\widetilde{M}, \widetilde{C})$ is injective, and hence $(M, C)$ is Kleinian. In this case, especially $\mathfrak{m}_{\mathrm{ADM}}\left(\widetilde{g}_{A F}\right)=0$. Therefore, it is enough to show that $\mathfrak{m}_{\mathrm{ADM}}\left(\widetilde{g}_{A F}\right) \geq 0$.

By combining [7, Theorem 8.1] (cf. 9]) with $Y(M, C)>0$ (replacing $M$ by its orientable double covering if necessary), $M$ can be decomposed uniquely into prime closed 3-manifolds

$$
M=N_{1} \# \cdots \# N_{\ell_{1}} \# \ell_{2}\left(S^{1} \times S^{2}\right),
$$

where $\pi_{1}\left(N_{i}\right)$ is finite for $i=1, \ldots, \ell_{1}$ and $\ell_{1}, \ell_{2}$ are nonnegative integers. By applying the $C$-prime decomposition theorem for closed 3-manifolds with flat conformal structures 10, 11 to $(M, C)$, there exists a flat conformal structure $C_{i}$ on each $N_{i}\left(i=1, \ldots, \ell_{1}\right)$. Then, Kuiper's Theorem [14] again implies that each $\left(N_{i}, C_{i}\right)$ is a nontrivial quotient of $\left(S^{3}, C_{0}\right)$. After taking an appropriate finite covering $M^{\prime}$ of $M$, we have

$$
M^{\prime}=\# \ell\left(S^{1} \times S^{2}\right) \quad \text { for some } \ell \geq 1 .
$$

Recall that $\widetilde{M}$ is the infinite universal covering of $M$. Then, there exists (uniquely) an infinite universal covering $\widetilde{M} \rightarrow M^{\prime}$. Moreover, since $\pi_{1}\left(M^{\prime}\right)$ is a finitely generated free group, it has a descending chain of finite index subgroups tending to $\pi_{1}(\widetilde{M})=\{e\}$. Let $g^{\prime}$ be the lifting of $g$ to $M^{\prime}$. Applying Proposition 2.1 to the normal infinite Riemannian covering $(\widetilde{M}, \widetilde{g}) \rightarrow\left(M^{\prime}, g^{\prime}\right)$, we have that

$$
\mathfrak{m}_{\mathrm{ADM}}\left(\widetilde{g}_{A F}\right) \geq 0 \text {. }
$$

This completes the proof of Theorem 1.1

Remark 3.1. Even if we replace the positivity $Y(M, C)>0$ in Theorem 1.1 by the nonnegativity $Y(M, C) \geq 0$, it seems that the same conclusion still holds. More precisely, we propose the following (cf. [5, 13]).

Conjecture. Let $M$ be a closed 3-manifold with flat conformal structure $C$. If its Yamabe constant is zero, then either (1) or (2) holds:

(1) There exists a flat metric $\bar{g} \in C$.

(2) There exists a smooth family $\left\{g_{t}\right\}_{0 \leq t \leq 1}$ of locally conformally flat metrics on $M$ such that $g_{0} \in C$ and $Y\left(M,\left[g_{1}\right]\right)>0$ (possibly $Y\left(M,\left[g_{t}\right]\right)<0$ for some $t \in(0,1))$.

In the case $(1)$, the universal covering $(\widetilde{M}, \widetilde{C})$ of $(M, C)$ is conformal to $\left(S^{3}-\right.$ $\left.\left\{p_{N}\right\}, C_{0}\right)$ where $p_{N}:=(1,0,0,0) \in S^{3}$, and hence $(M, C)$ is Kleinian. In the case (2), Theorem 1.1 implies that $\left(M,\left[g_{1}\right]\right)$ is Kleinian. The argument in the proof of 
Theorem 1.1 also implies that there exists a torsion free subgroup $\Gamma$ of finite index in $\pi_{1}(M)$ such that $\Gamma$ is either a trivial group or a nontrivial finitely generated free group. Then, the virtual cohomological dimension $\operatorname{vcd} \pi_{1}(M)$ of $\pi_{1}(M)$ is either 0 or 1 (see [6]). Therefore, $\left(M,\left[g_{1}\right]\right)$ is a closed Kleinian 3-manifold with vcd $\pi_{1}(M)<3$. The quasiconformal stability of Kleinian groups [12, Theorem 2] implies that any flat conformal structure on $M$ which is a smooth deformation of $\left[g_{1}\right]$ is also Kleinian; in particular $C$ is too.

\section{ACKNOWLEDGEMENTS}

The second author would like to thank Hiroyasu Izeki and Gilles Carron for helpful discussions and for useful comments respectively. The authors would like to thank the referee for crucial comments.

\section{REFERENCES}

1. K. Akutagawa, Aubin's lemma for the Yamabe constants of infinite coverings and a positive mass theorem, to appear in Math. Ann.

2. T. Aubin, Équations différentielles non linéaires et problème de Yamabe concernant la courbure scalaire, J. Math. Pures Appl. 55 (1976), 269-296. MR0431287 (55:4288)

3. T. Aubin, Some Nonlinear Problems in Riemannian Geometry, Springer Monographs in Mathematics, Springer, 1998. MR1636569 (99i:58001)

4. R. Bartnik, The mass of an asymptotically flat manifold, Comm. Pure Appl. Math. 39 (1986), 661-693. MR849427 (88b:58144)

5. J. P. Bourguignon, Une stratification de l'espace des structures riemanniennes, Compositio Math. 30 (1975), 1-41. MR0418147 (54:6189)

6. K. S. Brown, Cohomology of Groups, Graduate Texts in Math. 87, Springer, 1982. MR672956 $(83 \mathrm{k}: 20002)$

7. M. Gromov and H. B. Lawson Jr., Positive scalar curvature and the Dirac operator on complete Riemannian manifolds, Inst. Hautes Études Sci. Publ. Math. 58 (1983), 83-196. MR720933 (85g:58082)

8. M. Gromov, H. B. Lawson Jr. and W. Thurston, Hyperbolic 4-manifolds and conformally flat 3-manifolds, Inst. Hautes Études Sci. Publ. Math. 68 (1988), 27-45. MR1001446 (90k:57021)

9. J. Hempel, 3-Manifolds, Ann. of Math. Studies 86, Princeton Univ. Press, 1976. MR0415619 $(54: 3702)$

10. H. Izeki, On the decomposition of conformally flat manifolds, J. Math. Soc. Japan 45 (1993), 105-119. MR:1195686 (93h:53036)

11. H. Izeki, A deformation of flat conformal structures, Trans. Amer. Math. Soc. 348 (1996), 4939-4964. MR 1348862 (97c:53020)

12. H. Izeki, Quasiconformal stability of Kleinian groups and an embedding of a space of flat conformal structures, Conform. Geom. Dyn. 4 (2000), 108-119. MR.1799652 (2002a:57056)

13. O. Kobayashi, The scalar curvature of a metric with unit volume, Math. Ann. 279 (1987), 253-265. MR919505 (89a:53048)

14. N. H. Kuiper, On conformally flat spaces in the large, Ann. of Math. (2) 50 (1949), 916-924. MR0031310 (11:133b)

15. J. Lee and T. Parker, The Yamabe problem, Bull. Amer. Math. Soc. 17 (1987), 37-81. MR888880 (88f:53001)

16. T. Parker and C. Taubes, On Witten's proof of the positive energy theorem, Comm. Math. Phys. 84 (1982), 223-238. MR661134 (83m:83020)

17. R. Schoen, Conformal deformation of a Riemannian metric to constant scalar curvature, J. Differential Geom. 20 (1984), 479-495. MR788292 (86i:58137)

18. R. Schoen, Variational theory for the total scalar curvature functional for Riemannian metrics and related topics, Topics in Calculus of Variations, Lect. Notes in Math., vol. 1365, Springer, 1989, pp. 121-154. MR994021 (90g:58023)

19. R. Schoen and S.-T. Yau, Conformally flat manifolds, Kleinian groups and scalar curvature, Invent. Math. 92 (1988), 47-71. MR931204 (89c:58139) 
20. N. Trudinger, Remarks concerning the conformal deformation of Riemannian structures on compact manifolds, Ann. Scuola Norm. Sup. Pisa 22 (1968), 265-274. MR0240748 (39:2093)

21. E. Witten, A simple proof of the positive energy theorem, Comm. Math. Phys. 80 (1981), 381-402. MR626707 (83e:83035)

22. H. Yamabe, On a deformation of Riemannian structures on compact manifolds, Osaka J. Math. 12 (1960), 21-37. MR0125546 (23:A2847)

Department of Mathematics, University of Tsukuba, Tsukuba 305-8571, Japan

E-mail address: aiyama@math.tsukuba.ac.jp

Division of Mathematics, Graduate School of Information Sciences, Tohoku UniverSITY, SENDAI 980-8579, JAPAN

E-mail address: akutagawa@math.is.tohoku.ac.jp 